\title{
EI Uso De La Técnica "Lo Que Sé, Lo Que Quiero Aprender, Y Lo Que Aprendí”" Para Mejorar EI Nivel Literal De Comprensión Lectora En Inglés
}

\author{
Silvia Nataly Bejarano Criollo \\ Magíster en Lingüística y Didáctica de la Enseñanza de Idiomas Extranjeros \\ Docente de la Escuela Superior Politécnica de Chimborazo, \\ Facultad de Administración de Empresas, Ecuador \\ Cristina Paola Chamorro Ortega \\ Magíster en Lingüística y Didáctica de la Enseñanza de Idiomas Extranjeros \\ Docente de la Escuela Superior Politécnica de Chimborazo, \\ Facultad de Recursos Naturales, Ecuador

\section{Martha Lucía Lara Freire} \\ Magíster en Lingüística aplicada al Aprendizaje del Inglés \\ Docente de la Escuela Superior Politécnica de Chimborazo, \\ Facultad de Salud Pública, Ecuador

\section{Wilson Gonzalo Rojas Yumisaca} \\ Magister en Educación a Distancia y Abierta \\ Docente de la Escuela Superior Politécnica de Chimborazo, \\ Facultad de Administración de Empresas, Ecuador
}

\section{Doi:10.19044/esj.2019.v15n14p55 ～URL:http://dx.doi.org/10.19044/esj.2019.v15n14p55}

\section{Resumen}

El objetivo de la presente investigación fue mejorar el nivel literal de comprensión lectora por medio del uso de la técnica "lo que sé, lo que quiero saber, y lo que aprendí". Esta investigación cuali-cuantitativa de diseño cuasiexperimental estuvo conformada por dos grupos de trabajo, un grupo experimental y un grupo de control, ambos grupos con el mismo número de población, treinta estudiantes. Los estudiantes del grupo experimental participaron en una intervención de seis semanas, mientras que los estudiantes del grupo de control desarrollaron sus actividades de clase de manera tradicional, sin el uso de la técnica mencionada. Además, para la intervención se diseñaron doce planes de lección considerando temas incluidos en el Sílabo Institucional para Quinto Nivel, para lo cual se seleccionaron lecturas de diferentes fuentes considerando el nivel A1 del Marco Común Europeo de Referencia para Lenguas. Los instrumentos de evaluación y recolección de datos considerados fueron pre-test y post-test. Con los datos obtenidos de 
ambos grupos se pudieron analizar e interpretar los resultados mediante el estadístico puntaje $\mathrm{Z}$, a fin de comprobar la hipótesis alternativa y rechazar la hipótesis nula. Finalmente, se concluyó que la implementación de la técnica "lo que sé, lo que quiero saber, y lo que aprendí" mejora el nivel literal de la comprensión lectora.

Palabras-claves: Conocimiento previo, Diseño cuasiexperimental, Grupo experimental, Investigación cualitativa - cuantitativa, Puntaje Z.

\title{
The Use of the Technique "What I Know, What I Want to Know, and What I Learned" to Improve the Literal Level of Reading Comprehension in English
}

\section{Silvia Nataly Bejarano Criollo}

Magíster en Lingüística y Didáctica de la Enseñanza de Idiomas Extranjeros

Docente de la Escuela Superior Politécnica de Chimborazo,

Facultad de Administración de Empresas, Ecuador

\section{Cristina Paola Chamorro Ortega}

Magíster en Lingüística y Didáctica de la Enseñanza de Idiomas Extranjeros

Docente de la Escuela Superior Politécnica de Chimborazo,

Facultad de Recursos Naturales, Ecuador

\section{Martha Lucía Lara Freire}

Magíster en Lingüística aplicada al Aprendizaje del Inglés

Docente de la Escuela Superior Politécnica de Chimborazo,

Facultad de Salud Pública, Ecuador

Wilson Gonzalo Rojas Yumisaca

Magister en Educación a Distancia y Abierta

Docente de la Escuela Superior Politécnica de Chimborazo,

Facultad de Administración de Empresas, Ecuador

\begin{abstract}
The objective of this research was to improve the literal reading comprehension level by using the "what I know, what I want to know, and what I learned" technique. This quali-quantitative, quasi-experimental research considered two groups; an experimental and a control group, both with thirty students. The experimental group participated in a 6-week intervention, while the control group developed their class activities with the
\end{abstract}


traditional method without the application of the mentioned technique. In addition, for the intervention twelve lessons were created with topics included in Level V institutional syllabus, adapting articles from different sources and considering the A1 level from the Common European Framework of Reference for Languages. The evaluation and data collection instruments were pre-test and post-test. The obtained results of both groups were analyzed and interpreted by using the statistic ZTEST to prove the research hypothesis and reject the null hypothesis. In conclusion, the "what I know, what I want to know, and what I learned" technique improved the literal reading comprehension level.

Keywords: Previous knowledge, Quasi-experimental design, Experimental group, Qualitative - quantitative research, ZTEST.

\section{Introducción}

Es incuestionable la influencia que el idioma inglés tiene en la sociedad actual. Para muchas personas es un requisito primordial para conseguir un trabajo o continuar con sus estudios, pues a nivel mundial dicho idioma se ha establecido como una lengua franca (Lusting, 2018). La importancia que el idioma inglés posee en muchos países en diferentes áreas principalmente en la educación es evidente, es así que las políticas en instituciones educativas han sido modificadas con el fin de potenciar la enseñanza de este idioma acorde a las necesidades de los estudiantes para lograr un aprendizaje de calidad. Es preciso reconocer los ajustes que las universidades del país han venido haciendo en los últimos años dentro del proceso educativo al incluir el idioma inglés en los currículos universitarios.

Así mismo, el idioma inglés se ha convertido en uno de los requisitos fundamentales para los estudiantes que necesitan culminar sus estudios universitarios. Por ser un requisito obligatorio su aprendizaje ha sido complejo para muchos estudiantes, sea por falta de motivación por parte de los docentes, interés personal o porque el idioma inglés no fue considerado en colegios o escuelas donde estudiaron anteriormente. Todos estos factores han provocado un aprendizaje limitado y poco productivo en el proceso educativo.

La lectura es un proceso interactivo de comunicación establecida entre el texto y el lector. Su práctica misma se convierte en una actividad fundamental en la formación del ser humano por sus múltiples beneficios reflejados en la adquisición de nuevo vocabulario. Por otra parte, actúa como una acción inclusiva, fomenta la imaginación y permite el mejoramiento de los niveles de comprensión lectora existentes. Su práctica misma permite entender de forma clara y concisa las ideas que el autor quiere expresar en su escrito (Cuñachi \& Leyva, 2018). 
Calaméo (2018) afirma que "leer es comprender" y eso implica entender y descubrir lo que el lector quiere transmitir. Para lograr dicha comprensión es necesario ejercitar distintos niveles de comprensión lectora y uno de ellos es el nivel literal. En este nivel fundamental el lector expondrá su comprensión al extraer información clave, reconocer datos, hechos y detalles del texto. Así también, para que la comprensión del texto tenga el éxito deseado, el lector aplica procesos fundamentales como la observación, comparación, relación, clasificación, orden, entre otras (Calaméo, 2018).

Por lo anteriormente expuesto, los docentes del Centro de Idiomas ante la necesidad urgente de mejorar el aprendizaje del idioma inglés, consideraron fortalecer una de las estrategias básicas como es la lectura, a través de la implementación de la técnica S.Q.A (lo que se, lo que quiero saber, y lo que aprendí) para mejorar el nivel literal de comprensión lectora en los estudiantes. Por tanto, la presente investigación tuvo como objetivo analizar la incidencia del uso de la técnica S.Q.A en el mejoramiento del nivel literal de comprensión lectora en inglés en los estudiantes de Quinto Nivel del Centro de Idiomas de la Escuela Superior Politécnica de Chimborazo.

La técnica S.Q.A es una estrategia dinámica para guiar a los estudiantes antes, durante y después del proceso de lectura. Esta estrategia permite a los estudiantes plasmar ideas ya conocidas sobre el tema, esto se hace en la columna S, en la columna Q se puede expresar a través de preguntas lo que se quiere aprender sobre el tema y finalmente luego de desarrollar el proceso de lectura del texto, responder las preguntas y escribir la nueva información adquirida en la columna A. Esta estrategia de lectura es muy útil para fortalecer el nivel literal de comprensión lectora en los estudiantes, pues por su aplicación hace que los conocimientos previos del tema se activen y también incentiva el interés por la lectura. De igual manera, permite tener una mayor comprensión del significado de palabras nuevas en el texto, apropiarse del contenido y fortalecer la capacidad de entendimiento del mismo.

Como lo menciona Morales (2018) en su investigación titulada "Aplicación de la estrategia S.Q.A para mejorar el nivel de comprensión lectora de textos informativos de los estudiantes del tercer año de Educación Secundaria de la I.E. Jorge Martorell Flores" en Tacna, Perú; la aplicación de la estrategia S.Q.A logra mejorar el nivel literal de comprensión lectora del grupo experimental de manera significativa en comparación con el grupo de control. La estrategia S.Q.A permite activar los conocimientos previos y relacionarlos con los conocimientos adquiridos al interactuar con el texto y de esa manera permite a los estudiantes desarrollar sus capacidades en el nivel literal de comprensión lectora. Por otra parte, luego de analizar los resultados obtenidos, la autora recomienda aplicar la estrategia S.Q.A a fin de mejorar el nivel de comprensión lectora en los estudiantes. Además, menciona que la estrategia no sólo se utiliza para la comprensión de textos informativos, sino también 
para realizar diferentes trabajos de campo, trabajos de investigación $\mathrm{y}$ documentales (Morales, 2018).

\section{Importancia del Problema}

América Latina se ubica 2,34 puntos por debajo del promedio mundial en el English First English Proficiency Index (EF EPI) en todos los grupos de edad, siendo un fuerte indicador de que los sistemas educativos en América Latina tienen un desempeño menor en enseñanza de inglés que los de Asia y Europa. Ningún país en América Latina se ubica en la banda de dominio muy alto. Las puntuaciones registradas en el 2016 indican un descenso en nueve de los catorce países latinoamericanos incluidos en el estudio; tres países Ecuador, Guatemala y Perú, muestran una disminución de más de dos puntos (ACE, 2018).

En el Ecuador, inglés es el idioma extranjero que oficialmente se imparte en establecimientos educativos a nivel nacional. En este sentido, el Ministerio de Educación del Ecuador, implementó varias medidas enfocadas a mejorar la enseñanza-aprendizaje de la lengua inglesa en las instituciones educativas, una de estas medidas es la obligatoriedad de la enseñanza del inglés y la implementación del proyecto de Fortalecimiento de la Enseñanza del inglés. Sin embargo, a pesar de los esfuerzos realizados desde los años noventa, únicamente un mínimo de estudiantes del Bachillerato General Unificado logra alcanzar los niveles deseados de suficiencia del idioma inglés al culminar sus estudios de secundaria. De igual manera, la mayoría de los estudiantes universitarios presentan problemas para lograr obtener la acreditación de suficiencia respectiva, lo cual es un requisito para la graduación. Esta problemática también se refleja en la dificultad de los estudiantes para acceder a becas internacionales o inclusive para continuar sus estudios de posgrado (Lara, 2018).

La universidad ecuatoriana en los últimos tiempos ha tenido una participación relevante en la planificación de las acciones destinadas a manejar el desarrollo socio-económico de los pueblos. Pero, se considera que los programas ejecutados concernientes a la enseñanza-aprendizaje del idioma inglés hasta el momento, no han sido fructíferos para lograr las competencias necesarias.

Es así que, se ha podido identificar que los estudiantes demuestran limitada fluidez en la expresión oral y escrita, dificultad al interpretar textos en inglés y que no se aplica la lectura como hábito educativo en el proceso de enseñanza aprendizaje. Estas problemáticas se dan porque no existe la habilidad comprensiva en el idioma inglés para la comunicación; a pesar de que, una de las destrezas de mayor trabajo en la enseñanza de este idioma es la comprensión lectora. 
Los estudiantes no han desarrollado la capacidad de captar el significado de lo que se lee, formar asociaciones y prever la secuencia de ideas. También presentan inconvenientes al momento de asociar, relacionar y organizar ideas que el autor desea transmitir en el texto; captar el significado de oraciones y párrafos en relación con el contexto, reconocer su importancia relativa y la capacidad para leer con razonable velocidad.

Desafortunadamente, en el Ecuador no existe una cultura de lectura en el idioma inglés y se debe al estancamiento de los programas de enseñanza, cuyos referentes no van más allá de la gramática, la escasa lectura y escritura, la escasa motivación y el no reconocimiento del inglés como exigencia profesional (Velasteguí, Sánchez, \& Ramos, 2018).

Victoria (2019) manifiesta que existen diversos problemas en el proceso de enseñanza-aprendizaje de la comprensión lectora como falta de preocupación por parte de los docentes y directivos por encontrar métodos de estudios que posibiliten a los estudiantes comprender los textos escritos de manera más eficiente. Los estudiantes no sienten la necesidad de aprender o buscar información, ni de consultar textos especializados; por lo que, presentan limitaciones para argumentar sus aportaciones o criterios de los temas de estudio. Adicionalmente, se muestra un bajo nivel de hábito de lectura; lo cual, imposibilita el desarrollo de la comprensión lectora. Otro factor influyente es el desconocimiento de estrategias metodológicas en la aplicación del proceso lector, lo cual resulta en un bajo rendimiento académico (Victoria, 2019).

Por consiguiente, frente al problema de bajo nivel de comprensión lectora de los estudiantes de inglés como Lengua Extranjera, es indispensable desarrollar un estudio encaminado a mejorar el nivel de comprensión lectora literal en inglés de los estudiantes de Quinto Nivel, del Centro de Idiomas, de la Escuela Superior Politécnica de Chimborazo.

\section{Objetivos}

\section{Objetivo General}

- Mejorar el nivel literal de comprensión lectora por medio del uso de la técnica S.Q.A.

\section{Objetivos Específicos}

- Enfatizar el desarrollo de actividades de pre-lectura para mejorar el nivel de comprensión lectora.

- Activar el conocimiento previo de los estudiantes utilizando la técnica S.Q.A.

- Motivar la lectura a través de temas de interés para los estudiantes. 


\section{Hipótesis}

Hipótesis nula $\left(\mathbf{H}_{\mathbf{0}}\right)$ : Los estudiantes NO mejoran su habilidad de comprensión lectora literal por medio del uso de la técnica S.Q.A.

Hipótesis alterna $\left(\mathbf{H}_{1}\right)$ : Los estudiantes SI mejoran su habilidad de comprensión lectora literal por medio del uso de la técnica S.Q.A.

\section{Metodología}

\section{Método}

El método implica un proceso de ordenamiento, la dirección del pensamiento y de la acción para lograr algo previamente determinado; significa entonces, que un buen método será aquel que garantice un máximo aprovechamiento en la comprensión lectora en menos tiempo y con menos esfuerzo, por ello se utilizó el método inductivo-deductivo ya que el estudiante interactúa con el texto, lo asimila y lo comprende.

En la comprensión lectora intervienen procesos cognitivos, perceptivos y lingüísticos; por lo que, se puede decir que un buen lector posee dos tipos de habilidades: cognitivas y meta-cognitivas; que son las que permiten al lector tener conciencia de su proceso de comprensión y controlarlo a través de actividades de planificación, supervisión y evaluación del texto (Morales L. , 2018).

En el contexto del aprendizaje del inglés como lengua extranjera, la habilidad de combinar el conocimiento previo con el nuevo, juega un papel preponderante en el desarrollo del nivel de comprensión literal; por tanto, es responsabilidad del docente proporcionar el ambiente, estrategias, técnicas y materiales necesarios para que el estudiante pueda mejorar dicha habilidad (Mirman, 2018).

Este trabajo está enfocado en motivar a los estudiantes a leer a través de la aplicación de la técnica de lectura S.Q.A (lo que sé, lo que quiero saber, y lo que aprendí). Esta técnica se usa para guiar a los estudiantes antes de leer, con el fin de mejorar su nivel literal de comprensión lectora y, por ende, su rendimiento académico. También, la técnica está directamente relacionada con buenos desempeños académicos, pues es considerada como una herramienta para aprender a comprender textos; ya que, a mayor interacción con la lectura, la adquisición de contenidos será más evidentes (Arias, 2018).

\section{Materiales}

Para la intervención, se utilizaron planes de lección, hojas de trabajo y actividades enfocadas al desarrollo de la habilidad de comprensión lectora. Las lecturas se seleccionaron considerando el nivel A1 del Marco Común Europeo de Referencias para Lenguas y fueron adaptadas de diferentes fuentes como: PsyAlive, The Mofet Institute, The British Council y Linguapress. 


\section{Población}

La investigación se llevó a cabo en el Centro de Idiomas de la Escuela Superior Politécnica de Chimborazo de la ciudad de Riobamba, Ecuador, para lo cual se trabajó con una muestra de sesenta estudiantes de quinto nivel de inglés, quienes participaron en una intervención de cinco unidades durante seis semanas, con dos sesiones por semana en un tiempo estimado de 120 minutos, con la finalidad de mejorar su nivel literal de comprensión lectora.

\section{Enfoque}

La presente investigación tiene un enfoque cuali-cuantitativo, un diseño cuasi-experimental, y un nivel explicativo y descriptivo-correlacional; puesto que compara la relación de dos grupos de trabajo, un grupo experimental y un grupo de control.

El grupo de control constó de treinta estudiantes, quienes desarrollaron sus actividades de clase de manera tradicional, sin la aplicación de la técnica S.Q.A. Mientras que el grupo experimental estuvo sujeto a intervención y se aplicaron las hojas de trabajo y las actividades previamente elaboradas.

Los instrumentos de evaluación y recolección de datos fueron pre-test y post-test. El pre-test presentó un texto de 428 palabras titulado "Caffeine", compuesto por 4 párrafos, así como también 8 ítems enfocados al nivel literal de comprensión lectora, el mismo que fue aplicado a los dos grupos de estudio.

Por otro lado, el post-test estuvo compuesto por 6 párrafos, 649 palabras y 8 ítems, de igual manera se aplicó a los dos grupos a fin de medir la incidencia y grado de variación de la comprensión lectora.

\section{Validez y confiabilidad}

Los instrumentos fueron sometidos a una validación por 3 expertos en la enseñanza del Idioma Inglés, para lo cual se utilizó la tabla de validación de contenidos del instrumento y los criterios de evaluación.

Así también, para obtener el grado de confiabilidad de las pruebas pre-test y post- test se realizó una prueba piloto con la participación de 15 estudiantes que no formaron parte de la investigación, obteniendo un porcentaje de 0,85 de confiabilidad utilizando el coeficiente Alfa de Cronbach.

\section{Resultados}

Al finalizar la investigación, se analizaron e interpretaron los datos recopilados de los dos grupos involucrados. Para tabular el puntaje que obtuvieron los estudiantes en el pre-test y post-test se utilizaron tablas de Microsoft Excel. Mientras que, para la comprobación de la hipótesis se aplicó el estadístico puntaje Z, comprobando la efectividad de la técnica S.Q.A para mejorar el nivel literal de comprensión lectora en el proceso enseñanzaaprendizaje del idioma inglés. 
Gráfico 1. Comparación de resultados de respuestas en el nivel de lectura literal. Pre-test y Post-test del grupo de control

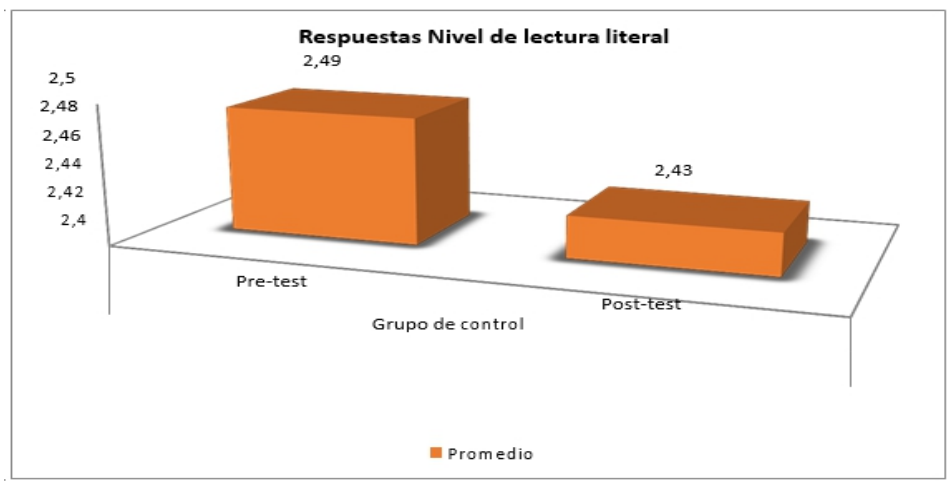

Fuente: Pre-test y Post-test del grupo de control Hecho por: Investigadores

El Gráfico 1, muestra los resultados de la evaluación inicial y final aplicada al grupo de control en cuanto al nivel literal de comprensión lectora, como se puede observar la nota obtenida por los estudiantes en el pre-test $(2,49 / 4,00)$ es mayor a la del post-test $(2,43 / 4,00)$. Esto indica, que los estudiantes del grupo de control iniciaron con un conocimiento medio de lectura literal y en lugar de mostrar mejoras, sus calificaciones fueron menores en el post-test, lo cual evidencia que debido al tiempo limitado de los docentes no se ha dado énfasis a la lectura en los estudiantes del Nivel V de inglés.

Gráfico 2. Comparación de resultados de respuestas en el nivel de lectura literal. Pre-test y Post-test del grupo experimental

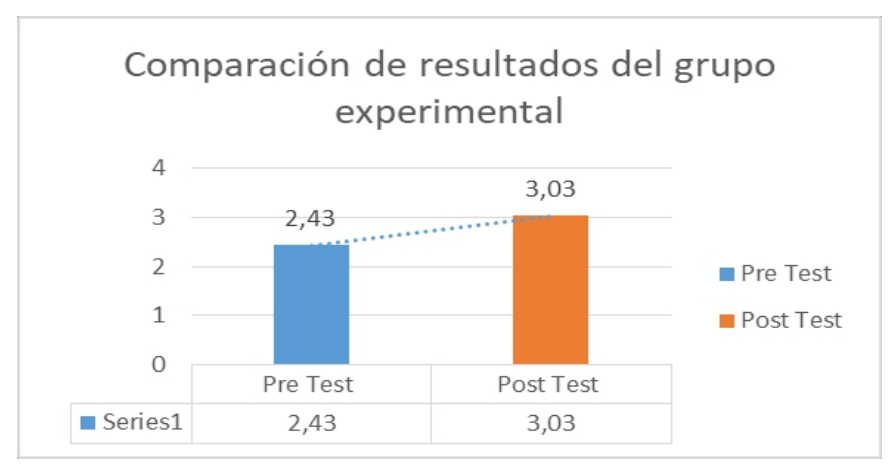

Fuente: Pre-test y Post-test del grupo experimental

Hecho por: Investigadores

El gráfico muestra que el promedio de las preguntas de comprensión lectora, nivel literal es de 2,43/4 en el pre-test y de 3,03/4 en el post-test, la diferencia en asertividad es de 0,6 . Se pudo observar que los estudiantes no solo mejoraron su habilidad de comprensión lectora, sino que también incrementó su interés por la lectura y se comprobó que la técnica 
implementada facilita el aprendizaje significativo durante el proceso de lectura comprensiva y ayuda al estudiante a internalizar sus conocimientos y cimentar su nuevo aprendizaje.

\section{Discusión}

Considerando que el aprendizaje del inglés como Lengua Extranjera ocupa un gran espacio en la educación, éste estudio estuvo enfocado a que los estudiantes usen la técnica de lectura S.Q.A, con el propósito de mejorar su nivel literal de comprensión lectora, lo cual proporcionó un rendimiento académico optimo ya que mientras más se lee; la adquisición de comprensión lectora se incrementa conjuntamente en el desarrollo del nivel de comprensión literal.

La intervención se realizó implementando el organizador grafico S.Q.A. Esta técnica tuvo una incidencia significativa en el aprendizaje de los estudiantes de Nivel V del Centro de Idiomas, de la Escuela Superior Politécnica de Chimborazo, como lo muestra el Gráfico 2, donde el promedio del nivel literal de comprensión lectora del grupo experimental es de 2,43/4,00 en el pre-test y en el post-test de 3,03/4,00.

Penagos (2018) en su investigación titulada "Las secuencias didácticas, como situaciones de aprendizaje para mejorar la comprensión lectora" sostiene que la técnica S.Q.A, es efectiva ya que a través de la misma el alumno puede expresar su comprensión sobre un tema y que lo puede realizar en tres pasos muy importantes: primero, qué sé; segundo qué quiero aprender; y tercero, qué aprendí; esto contextualizado al tema. Es decir, no se utilizaron métodos tradicionales de enseñanza que llevaban a la rutina, sino más bien se empleó esta técnica con la creatividad del docente para que el alumno aprenda de una forma proactiva, más no mecanizada.

En este estudio, es importante también destacar que la técnica S.Q.A despertó el interés de los estudiantes en las clases de inglés para desarrollar actividades de comprensión lectora; ya que los mismos participaron activamente en la revisión de su aprendizaje previo, para luego interesarse en lo que desean saber del tema y finalmente, reproducir lo que aprendieron sobre el tema (Penagos, 2018).

La diferencia entre este estudio y otros estudios se centra principalmente en los resultados obtenidos en el nivel literal de comprensión lectora. Al inicio de la intervención, los estudiantes se sentían desmotivados, ya que las actividades de lectura en inglés eran monótonas y se centraban mayormente en el uso de técnicas tradicionales, lo cual no contribuía a un desarrollo académico positivo. Teniendo en cuenta que, en las clases de inglés, el desarrollo de la comprensión lectora es importante para adquirir un L2 a través de diferentes actividades. 
Durante el plan de intervención, al dar mayor énfasis a la implementación de la técnica S.Q.A para actividades de comprensión lectora, se restó tiempo a las tres destrezas restantes del idioma inglés. Sin embargo, el uso selectivo de esta técnica colocó a los estudiantes en el mejor camino para un nuevo aprendizaje.

En síntesis, existen cientos de métodos, herramientas o técnicas que influyen en el mejoramiento de la habilidad de comprensión lectora de los estudiantes, pero la técnica S.Q.A ayudó a transformar la enseñanzaaprendizaje de la comprensión lectora literal de los estudiantes del Nivel V del Centro de Idiomas de la Escuela Superior Politécnica de Chimborazo.

\section{Conclusion}

Con los resultados expuestos en la presente investigación, observamos que el nivel literal de comprensión lectora por medio del uso de la técnica S.Q.A si mejora. Así, hemos podido constatar que los estudiantes del grupo experimental, quienes formaron parte de la intervención han obtenido un promedio de 3,03 en el post-test, mientras que los estudiantes del grupo de control que no fueron parten de la intervención alcanzaron un promedio de 2,43 , en cuanto al nivel literal de comprensión lectora. Esto demuestra que la diferencia de asertividad es de 0,6.

Esta investigación también constató la importancia de desarrollar actividades de pre-lectura tales como revisión de palabras nuevas, intercambio de ideas en grupos de trabajo colaborativo y la utilización del organizador gráfico S.Q.A; puesto que, estas actividades influyen directamente en el mejoramiento del nivel de comprensión lectora.

Dentro del análisis realizado acerca de la utilización del organizador gráfico S.Q.A, se demostró que esta técnica si activa el conocimiento previo de los estudiantes, ya que en la primera columna denominada "lo que sé", una vez que se ha expuesto el tema de lectura, el docente solicita a los estudiantes determinar lo que saben acerca del mismo.

Finalmente, se debe seleccionar lecturas tomando en cuenta la edad de los estudiantes, el nivel y temas que sean de su interés, de esa manera se despierta la motivación por la lectura lo que permite obtener el mayor provecho de las actividades planificadas para las clases de inglés. No obstante, se observó que algunos estudiantes desarrollaron las actividades de lectura más por obligación que por gusto; en consecuencia, la falta de hábito por la lectura disminuyó la posibilidad de mejorar el nivel literal de comprensión lectora.

\section{Referencias}

1. ACE. (2 de Abril de 2018). An Istec Initiative. Obtenido de https://www.istec.org/ace/2018/04/02/panorama-del-aprendizaje-delidioma-ingles-en-latinoamerica/ 
2. Arias, M. d. (2018). La interrogación de textos como estratégia para desarrollar la comprensión lectora. San Salvador: Universidad Peruana.

3. Calaméo. (28 de Abril de 2018). Calaméo. Obtenido de https://es.calameo.com/books/00101312881a495f61954

4. Cuñachi, G., \& Leyva, G. (2018). Comprensión Lectora y el aprendizaje en el área de Comunicación. La Cantuta: Universidad Nacional de Educación Enrique Guzmán y Valle.

5. Lara, F. (2018). Medios digitales como recursos digitales para fortalecer la enseñanza del idioma inglés. Loja: Universidad Nacional de Loja.

6. Lusting, R. (23 de Mayo de 2018). BBC . Obtenido de https://www.bbc.com/mundo/noticias-44232687

7. Mirman, A. (2018). La enseñanza y el aprendizaje del inglés como Lengua Extranjera. Sevilla: Universidad de Sevilla.

8. Morales, C. (22 de Abril de 2018). Repositorio Insititucional Digital. Obtenido de repositorio.unjbg.edu.pe/handle/UNJBG/2771?show=full

9. Morales, L. (2018). Cognitive skills through the Cooperative Learning strategy. Scielo, 11.

10. Penagos, L. (2018). La secuencias didácticas, como situaciones de aprendizaje para mejorar la comprensión lectora. Ibagué-Colombia: Universidad de Tolima.

11. Velasteguí, E., Sánchez, S., \& Ramos, E. (2018). El hábito de la lectura y el disfrute de textos por medio de un tutorial multimedia. Ambato: Universidad Técnica de Ambato.

12. Victoria, R. (2019). La comprensión lectora en la enseñanza del español L1/L2 de la teoría a la práctica. Oxon: Routledge. 